\title{
Influencia del porcentaje de sustitución de emulsión asfáltica en frio en las propiedades físicas de cilindros de mortero y cilindros de tierra comprimida (CTC)
}

\author{
H. Cañola ${ }^{1 *}$, G. Barreto ${ }^{2}$, F. Granda-Ramírez ${ }^{3}$, A. Urrego ${ }^{4}$ \\ *Hernán Cañola: hernan.canola@colmayor.edu.co \\ ${ }^{1}$ Grupo de Investigación Ambiente, Hábitat y Sostenibilidad, Facultad de Arquitectura e Ingenierí,a Institución \\ Universitaria Colegio Mayor de Antioquia, Medellín, Colombia \\ ${ }^{2}$ Departamento de Gestión de la Construcción, Universidade Católica del Norte, Antafogasta, Chile \\ ${ }^{3}$ Grupo de Investigación Ambiente, Hábitat y Sostenibilidad, Facultad de Arquitectura e Ingenierí,a Institución \\ Universitaria Colegio Mayor de Antioquia, Medellín, Colombia \\ ${ }^{4}$ Grupo de Investigación Ambiente, Hábitat y Sostenibilidad, Facultad de Arquitectura e Ingenierí,a Institución \\ Universitaria Colegio Mayor de Antioquia, Medellín, Colombia
}

\begin{abstract}
Se estudiaron las variaciones de los Coeficientes de Absorción Capilar (CAC) y la Resistencia a la Penetración de Agua (RPA) en Cilindros de Tierra Comprimida (CTC) y Cilindros de Concreto (CC) adicionados con emulsiones asfálticas en frio. Las mezclas de los CC fueron adicionadas con porcentajes de emulsión entre el $10 \%$ y el $40 \%$ respecto al peso del cemento y para las mezclas de CTC se utilizó entre el $25 \%$ y el $100 \%$ respecto al peso del agua. Se lograron incrementos del 10241\% para RPA del CTC y de 4593\% para los CC; además el uso de la emulsión permitió una reducción del CAC del $91.4 \%$ para los CTC y del $97.5 \%$ para los CC, demostrando ser una modificación eficiente.
\end{abstract}

RESUMEN

Palabras clave: Cilindros de tierra comprimida; Cilindros de concreto; Correlaciones; Propiedades físicas; Emulsión asfáltica.

\begin{abstract}
Variations of Capillary Absorption Coefficients (CAC) and Resistance of Water Penetration (RWP) in Compressed Earth Cylinders (CEC) and Mortar Cylinders (CC) added with cold asphalt emulsions were studied. The CC mixtures were added with emulsion percentages between $10 \%$ and $40 \%$ respect to the weight of the cement and for the CEC mixtures between $25 \%$ and $100 \%$ respect to the weight of the water was used. Increases of $10241 \%$ were achieved for RWP of the CEC and 4593\% for the CC; Furthermore, the use of the emulsion allowed a reduction of the CAC of $91.4 \%$ for the CEC and $97.5 \%$ for the $\mathrm{CC}$, proving to be an efficient modification.
\end{abstract}

\section{RESUMO}

Foram estudadas as variações dos Coeficientes de Absorção Capilar (CAC) e da Resistência à Penetração de Água (RPA) em Cilindros de Terra Comprimida (CTC) e Cilindros de morteiro 
(CC) adicionados com emulsões asfálticas a frio. As misturas de CC foram adicionadas com percentagens de emulsão entre $10 \%$ e $40 \%$ em relação ao peso do cimento e para as misturas de CTC entre $25 \%$ e $100 \%$ em relação ao peso da água. Aumentos de $10241 \%$ foram alcançados para RPA do CTC e 4593\% para o CC; Além disso, o uso da emulsão permitiu uma redução do CAC de $91,4 \%$ para o CTC e 97,5\% para o CC, demonstrando ser uma modificação eficiente.

\section{INTRODUCCIÓN}

Se entiende como durabilidad en el área de la construcción, a la capacidad que presentan los elementos constructivos de conservar sus atributos físicos en el tiempo sin que se presenten mantenimientos prematuros o intervenciones constructivas no previstas, dependiendo siempre y cuando de las características físico-mecánicas y atributos físicos de los elementos constructivos con relación a su uso y condiciones ambientales (Broto and Soria, 2005; Monjo and Maldonado, 2001; Silva et al., 2017). Sin embargo, los sistemas constructivos como la mampostería para fachada (ladrillos, bloques de tierra comprimida, bloques de concreto), mampostería para sobrecimiento (bloques de concreto) y muros de medianería se encuentran sometidos a agentes externos como la lluvia y el agua proveniente del terreno que afectan la estabilidad física y estructural de estos elementos como consecuencia de la presencia de humedad durante largos periodos de tiempo (Jiménez, 2005; Anastasopoulos, 2013; Tokimatsu, 2012; Adam and Agib, 2001); Por otro lado (Cañola et al., 2020) determinan que el contacto de los sistemas de mampostería con agentes externos como el agua lluvia y el agua contenida en el terreno, pueden afectar los diferentes componentes de los muros como los mampuestos y morteros debido a la formación de agentes salinos correspondientes a las eflorescencias; por otra parte (Tsukagoshi et al., 2012; Saija, 1995) determinan que aunque los elementos constructivos como los morteros presenten sistemas de impermeabilización como barreras físicas en poliuretano frente a los agentes externos como el agua, estos elementos con el paso del tiempo pierden sus características físicas y espesor, haciendo que los sustratos en mortero sean mas vulnerables a los procesos de degradación por la carbonatación y procesos de agrietamiento. Es por lo anterior que (Othman et al., 2015; Shoemaker and House, 2006) determinan que el control y solución a las problemáticas de humedad en especial en edificaciones hospitalarias, es de vital importancia para garantizar la funcionalidad de las edificaciones y garantizar el confort de sus residentes. Como consecuencia del proceso de deterioro que sufren los morteros y los elementos constructivos debido a los agentes ambientales externos, (Falchi et al., 2013; Lanzón and García-Ruiz, 2009) determinaron que el uso de morteros con adiciones de puzolana-cal y Silano permite la mejo de las propiedades mecánicas de estos elementos y la repelencia al agua, lo que reduce las formaciones de eflorescencias. Teniendo presente que el agua contenida en el terrenos es considerada un agente degradante para los muros y morteros en las edificaciones, (Shoemaker and House, 2006) establecen que la humedad capilar es considerada como una de las principales causas de degradación en los sistemas de mampostería; la cual ocasiona procesos de condensación al interior de la edificación, cristalización de sales, corrosión, procesos de desprendimientos de acabados arquitectónicos, configura condiciones óptimas para el crecimiento de moho, afectar la salud y el confort de los residentes (Shoemaker and House, 2006; Carrer et al., 2015; Karagiannis et al., 2017).

Actualmente, el conocimiento con relación a la permeabilidad en los materiales de construcción ha cobrado relevancia debido a las problemáticas derivadas de la humedad presentes en sistemas constructivos como: revoques, estucos, mamposterías y demás acabados; estas problemáticas o lesiones patológicas debidas a la carencia de tratamientos preventivos, tratamientos correctivos y controles técnicos; por esta razón, la presencia incontrolada de humedad en los materiales de 
construcción ha sido considerada como uno de los factores responsables de la pérdida de durabilidad (Karagiannis et al., 2017). Se han desarrollado métodos para minimizar las problemáticas asociadas a la humedad mediante la implementación de sistemas impermeables externos como es el caso de barreras químicas (hidrorepelentes) o la inclusión de aditivos en las mezclas utilizadas en la fabricación de bloques de concreto y bloques de tierra comprimida entre otros. Pero ninguna de las alternativas anteriormente mencionadas han logrado desarrollar un mampuesto que presente total hidrorepelencia (Adam and Agib, 2001; Cañola and Echavarría 2017; Cañola et al., 2018; Pereira et al., 2018). Algunas investigaciones en las cuales se ha estudiado la problemática de humedad, sus efectos y la hidrorepelencia en muros se muestran a continuación:

Cho et al. (Cho et al., 2017) determinaron que el exceso de humedad en los concretos afecta las propiedades físicas del mismo como el módulo de elasticidad, contracción por secado, deformación y propiedades mecánicas como la resistencia a la compresión; además, determinaron que para predecir cuantitativamente el deterioro del concreto, es necesario identificar las condiciones ambientales en las cuales se encuentra expuesto el material y establecer el movimiento del aire y la velocidad de reacción del concreto con relación a la transferencia de calor y humedad. Así mismo, Muhammad et al. (Muhammad et al., 2015) establecieron que someter elementos constructivos de concreto a la penetración continua de agua y otros fluidos genera una reducción con relación a la durabilidad. Además, determinaron que la asociación entre el entorno y los componentes de constitución determinan la vida útil a largo plazo de los concretos. Por otro lado, Cui et al. (Cui et al., 2012) determinaron que el uso de cenizas volantes como alternativa al uso del cemento en los elementos constructivos a base de concreto pueden mejorar la impermeabilidad y estanqueidad hasta en un $50 \%$ con relación a las mezclas traiciónales; dicho estudio fue corroborado por Teng et al. (Teng et al., 2014).

También Litvan (Litvan, 1996) demostró que el uso de barreras físicas impermeables aumenta la durabilidad y longevidad de los elementos constructivos en concreto y proporciona protección al acero de refuerzo frente al ataque por carbonatación. Del mismo modo, Almusallam et al. (Almusallam et al., 2002) establecieron que el uso de resinas de poliuretano y epoxi superficiales son alternativas para la mejora de la permeabilidad, reducción de la penetración de cloruros, difusión y disminución del ataque de sulfatos en elementos de concreto en comparación a otros productos como agentes de recubrimiento. Sin embargo, Zhu et al (Zhu et al, 2013) y Muhammad et al. (Muhammad et al., 2015) establecen que el uso de aditivos en los elementos constructivos a base de concreto como el silano mejoran su durabilidad en comparación a los sistemas de impermeabilización superficial; sin embargo, el uso de estos aditivos presentan una reducción de las propiedades mecánicas del concreto como la resistencia a la compresión en un 10\%; al igual que el uso del silano. La implementación de emulsiones asfálticas como aditivo reductor de la permeabilidad en morteros o concretos también ocasiona una reducción de las propiedades mecánicas de estos elementos constructivos (Bołtryk and Małaszkiewicz, 2013). Además de los anteriores autores, Cañola et al. (Cañola et al., 2018) estudiaron el uso de compuestos bituminosos en Bloques de Tierra Comprimida (BTC), encontrando que a mayor uso de estos aditivos menores son las propiedades mecánicas, sin embargo, determinaron que los BTC sometidos a agentas externos como el agua presentan pérdidas de masa de un $8 \%$, pero las adiciones de compuestos bituminosos en un $25 \%$ en relación a la cantidad de agua minimizan esta problemática.

Basados en las problemáticas derivadas de la permeabilidad, para determinar la impermeabilidad de elementos constructivos es necesario estimar su coeficiente de absorción capilar, según Zürcher et al (Zürcher and Frank, 2014) los elementos constructivos con coeficientes menores a $0.030 \mathrm{~kg}$ $\mathrm{s}^{-1 / 2} \mathrm{~m}^{-2}$ son repelentes al agua y con valores menores a $0.008 \mathrm{~kg} \mathrm{~s}^{-1 / 2} \mathrm{~m}^{-2}$ son considerados hidrófugos. Debido a lo anterior Cañola et al. (Cañola and Echavarría, 2017) obtuvieron en su estudio de bloques de concreto con emulsión de parafina coeficientes de absorción capilar entre 
$0.076 \mathrm{~kg} \mathrm{~s}^{-1 / 2} \mathrm{~m}^{-2}$ y $0.005 \mathrm{~kg} \mathrm{~s}^{-1 / 2} \mathrm{~m}^{-2}$. También encontraron resistencias a la penetración de humedad entre 9.30E6 s m $\mathrm{s}^{-2}$ y $5.93 \mathrm{E} 7 \mathrm{~s} \mathrm{~m}^{-2}$. Por otro lado, Badogiannis et al. (Badogiannis et al., 2015) en su investigación sobre durabilidad de concretos autocompactantes con metacaolín determinaron velocidades de absorción capilar entre $1.30 \mathrm{E}-5 \mathrm{~m} \mathrm{~s}^{-1 / 2}$ y $3.10 \mathrm{E}-5 \mathrm{~m} \mathrm{~s}^{-1 / 2}$, del mismo modo Wilson et al. (Wilson et al., 1994) analizaron la velocidad de absorción en materiales porosos para la construcción como morteros, con valores comprendidos entre $8.90 \mathrm{E}-5 \mathrm{~m} \mathrm{~s}^{-1 / 2}$ y $1.86 \mathrm{E}-4 \mathrm{~m}$ $\mathrm{s}^{-1 / 2}$.

En diferentes investigaciones se han desarrollado tratamientos para la disminución de los coeficientes de adsorción capilar en diferentes materiales de construcción como BTC, morteros y bloques de concreto, pero no se ha generado una correlación del comportamiento de estos materiales entre sí con relación al uso de emulsiones asfálticas como aditivo reductor de la permeabilidad, es por lo anterior que en esta investigación se pretende establecer la variación de propiedades físicas como los coeficientes de adsorción capilar, porosidad y resistencia a la penetración de humedad entre muestras cilíndricas elaboras con mezclas para la fabricación de bloques de concreto y BTC.

\section{MATERIALES Y MÉTODOS}

Para el desarrollo de esta investigación se realizaron 100 probetas cilíndricas de cada una de las mezclas utilizadas en la fabricación de bloques de concreto y BTC, ambos adicionados con emulsión asfáltica en frío (Sika $\left.{ }^{\circledR}\right)$. Para la fabricación de las probetas fueron utilizados los siguientes materiales descritos en la Tabla 1, de los cuales se determinó su densidad por medio de correlaciones volumétricas y masas.

Tabla 1. Densidades de los materiales utilizados en la fabricación de cilindros de mortero y CTC.

\begin{tabular}{|c|c|}
\hline Materiales & Densidad $\left.\mathbf{( k g} / \mathbf{m}^{\mathbf{3}}\right)$ \\
\hline Arena de pega & 1600 \\
\hline Agua & 1000 \\
\hline Cemento Portland tipo 1 & 3150 \\
\hline Emulsión asfáltica & 1060 \\
\hline Tierra & 1390 \\
\hline
\end{tabular}

Las mezclas utilizadas para la fabricación de cilindros de mortero fueron adicionadas con emulsión asfáltica en frío con porcentajes de 10\%, 20\%, 30\% y 40\% respecto al peso del cemento y las mezclas utilizadas para la fabricación de cilindros en tierra compactada fueron elaboradas con porcentajes de emulsión asfáltica de $25 \%, 50 \%, 75 \%$ y $100 \%$, respecto al peso del agua.

El desarrollo experimental de la investigación se dividió en cuatro etapas: la primera consistió en la caracterización granulométrica del árido utilizado para fabricación de cilindros de mortero y CTC según la norma ASTM D422 (ASTM, 2007a), en la segunda etapa se estudió la succión capilar para evaluar el coeficiente de absorción capilar, la resistencia a la penetración del agua y la porosidad, lo anterior bajo parámetros de la norma ASTM C1585-04 (ASTM, 2007b) y la norma UNE PrUNE 83.982 (UNE, 2007), en la tercera y última etapa se realizó un análisis de correlación con la finalidad de verificar la influencia del porcentaje de sustitución de emulsión en el RPA (Resistencia a la penetración de agua) de CTC y de los cilindros de mortero. 


\section{RESULTADOS Y ANÁLISIS}

Para el análisis granulométrico de la tierra y la arena de pega se tomó una muestra de 2000 gramos por cada material a analizar, posteriormente, de cada muestra cuearteada, fue tomada una muestra de $500 \mathrm{~g}$, las cuales fueron secadas al horno durante 24 horas a una temperatura constante de $110^{\circ} \mathrm{C}$ $\pm 5^{\circ} \mathrm{C}$, una vez secas las muestras, estas fueron sometidas a un proceso de vibrado de manera independiente con una seria de tamices de diámetros variable en disposición vertical. Una vez analizados los pesos, se obtuvo los porcentajes de arena de pega y tierra retenidos y pasantes en cada tamiz, con lo cual se determinaron las curvas granulométricas y sus respectivos módulos de finura equivalentes a 2,98\% para la arena y de $1.80 \%$ para la tierra; a partir de lo anterior se estimó entonces que la arena corresponde a una arena media y la tierra a un suelo fino granular según la norma ASTM D422 (ASTM, 2007a) (Figura 1).

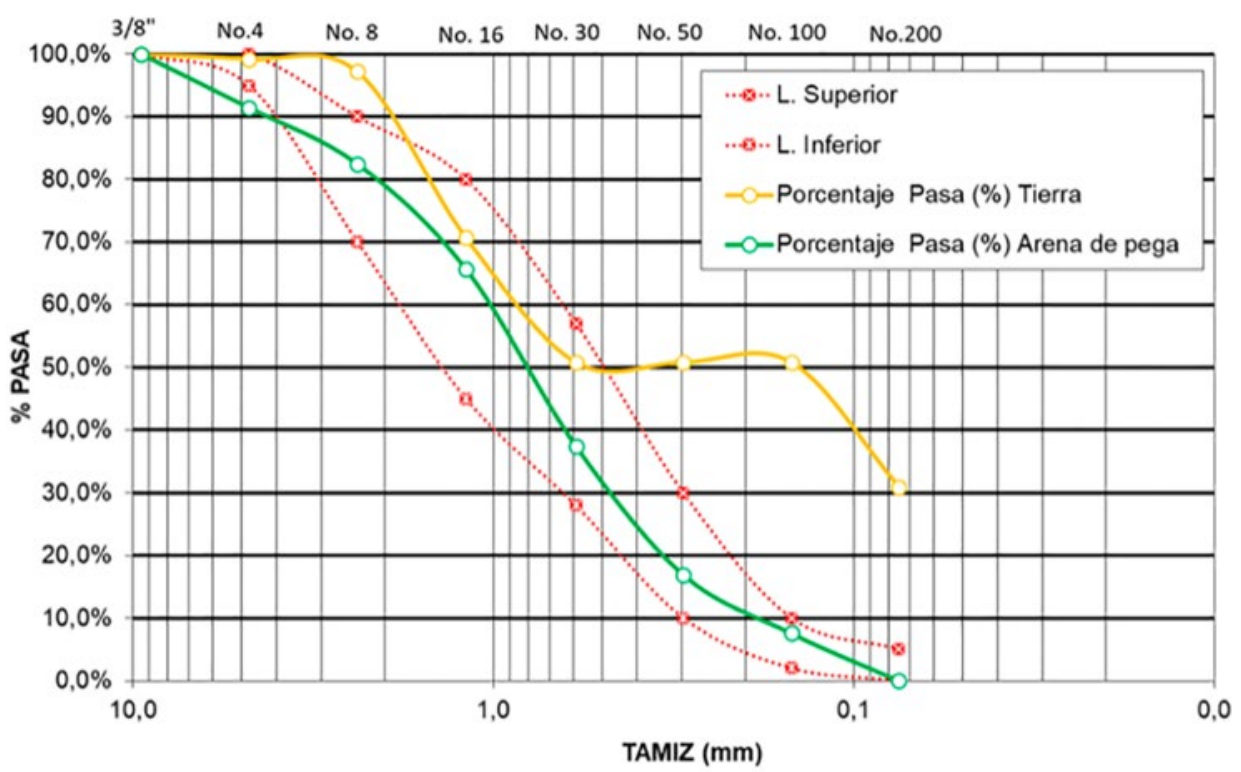

Figura 1. Curvas granulométricas de tierra y arena de pega.

Para la segunda etapa relacionada con el análisis de succión capilar bajo parámetros de las normas ASTM C1585-04 (ASTM, 2007b) y UNE PrUNE 83.982 (UNE, 2007) mediante el uso de probetas cilíndricas con radio de $0.05 \mathrm{~m}$ y altura de $0.05 \mathrm{~m}$, se determinaron el coeficiente de absorción capilar (1), la porosidad (2) y la resistencia a la penetración de humedad (3) de mezclas para CTC y cilindros de mortero (Figura 2 ).

$$
\begin{aligned}
& k=\frac{Q_{n}-Q_{0}}{\sqrt{t_{n}}} \frac{1}{A} \\
& \varepsilon=\frac{Q_{n}-Q_{0}}{A h \rho}
\end{aligned}
$$




$$
m=\frac{t_{n}}{h^{2}}
$$

Donde:

k: Coeficiente de absorción capilar.

$\mathrm{Q}_{\mathrm{n}}$ : Masa de la probeta en el punto crítico $(\mathrm{Kg})$.

$\mathrm{Q}_{0}$ : Masa de la probeta al inicio $(\mathrm{Kg})$.

$t_{n}$ : Tiempo en el punto crítico de saturación de la probeta (s).

A: Área de succión de la probeta $\left(\mathrm{m}^{2}\right)$.

$\varepsilon$ : Porosidad.

h: Altura o espesor total de la probeta $(\mathrm{m})$.

$\rho$ : Densidad del agua $\left(\mathrm{kg} \mathrm{m}^{-3}\right)$

A

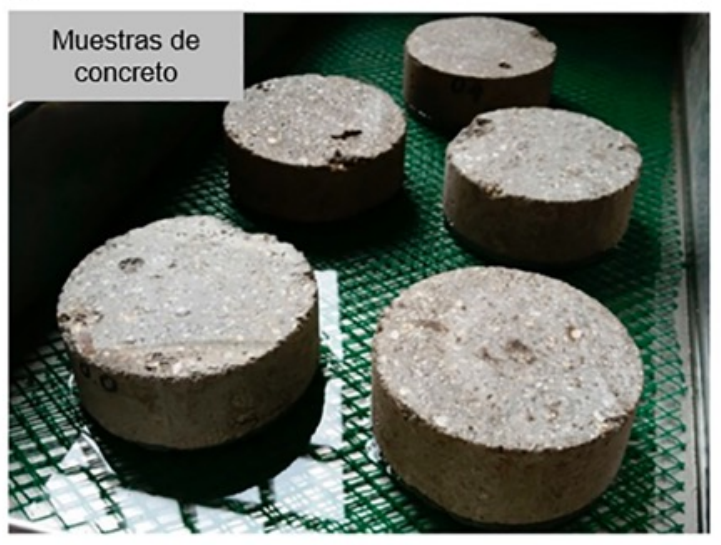

B

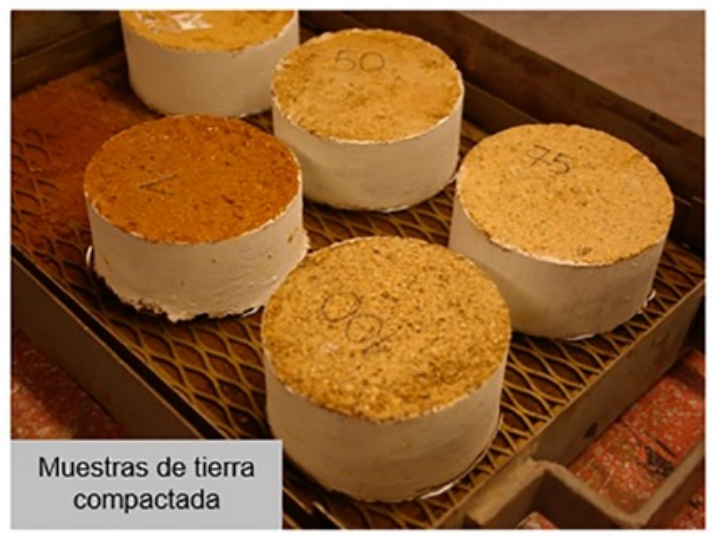

Figura 2. A) Muestra cilíndricas de mortero; B) Muestras cilíndricas de tierra compactada bajo análisis de succión capilar.

En la Tabla 2 se muestran los resultados de resistencia a la penetración, coeficiente de absorción capilar, y porosidad efectiva con relación a los porcentajes de sustitución de emulsión para el BTC. Tabla 2. Resultados sustitución de emulsión en CTC.

\begin{tabular}{|c|c|c|c|c|}
\hline Mezcla & $\begin{array}{c}\text { \% de } \\
\text { sustitución }\end{array}$ & $\begin{array}{c}\text { (RPA) Resistencia a la } \\
\text { penetración del agua } \\
\left(\mathbf{1 0}^{\mathbf{6}} \mathbf{s ~ m}^{-\mathbf{2}} \mathbf{~}\right.\end{array}$ & $\begin{array}{c}\text { (CAC) Coeficiente de } \\
\text { absorción capilar } \\
\left(\mathbf{k g ~ s}^{\mathbf{- 1 / 2}} \mathbf{~ m}^{\mathbf{- 2}} \mathbf{)}\right.\end{array}$ & $\begin{array}{c}\text { Porosidad } \\
\text { efectiva (\%) }\end{array}$ \\
\hline $0 \%$ emulsión & 0 & 1,56 & 0,233 & 29 \\
\hline $25 \%$ emulsión & 25 & 34,56 & 0,058 & 34 \\
\hline $50 \%$ emulsión & 50 & 127,81 & 0,027 & 30 \\
\hline $75 \%$ emulsión & 75 & 127,81 & 0,025 & 29 \\
\hline $\begin{array}{c}100 \% \\
\text { emulsión }\end{array}$ & 100 & 159,76 & 0,020 & 26 \\
\hline
\end{tabular}

De acuerdo a los resultados obtenidos (Tabla 2), se observa que a medida que fue aumentando el porcentaje de sustitución de emulsión, la resistencia a la penetración del agua aumento de forma gradual. En el caso del coeficiente de absorción capilar este tuvo una reducción a medida que iba aumentando el porcentaje de emulsión llegando a una reducción máxima del 91\% 
aproximadamente para la sustitución del 100\%. Finalmente, la porosidad efectiva no tuvo una variación representativa teniendo solo una reducción del 10\% en relación a la referencia.

En la Tabla 3 se muestran los resultados de resistencia a la penetración, coeficiente de absorción capilar y porosidad efectiva para los porcentajes de sustitución de emulsión para los cilindros de mortero.

Tabla 3. Resultados sustitución de emulsión para los cilindros de mortero.

\begin{tabular}{|c|c|c|c|c|}
\hline Mezcla & \% se sustitución & $\begin{array}{c}\text { (RPA) Resistencia a } \\
\text { la penetración del } \\
\text { agua } \\
\left(\mathbf{1 0}^{\mathbf{6}} \mathbf{s ~ m}^{-\mathbf{2}} \mathbf{)}\right.\end{array}$ & $\begin{array}{c}\text { (CAC) Coeficiente } \\
\text { de absorción capilar } \\
\left(\mathbf{k g ~ s}^{\mathbf{- 1 / 2}} \mathbf{~ m}^{\mathbf{2}} \mathbf{)}\right.\end{array}$ & $\begin{array}{c}\text { Porosidad } \\
\text { efectiva } \\
\mathbf{( \% )}\end{array}$ \\
\hline $0 \%$ emulsión & 0 & 9,29 & 0,081 & 0,247 \\
\hline $10 \%$ emulsión & 10 & 89,25 & 0,014 & 0,140 \\
\hline $20 \%$ emulsión & 20 & 133,88 & 0,005 & 0,060 \\
\hline $30 \%$ emulsión & 30 & 401,65 & 0,002 & 0,050 \\
\hline $40 \%$ emulsión & 40 & 426,66 & 0,002 & 0,045 \\
\hline
\end{tabular}

De acuerdo a los resultados obtenidos (Tabla 3), se observa que a medida que fue aumentando el porcentaje de sustitución de emulsión la resistencia a la penetración del agua aumento concordando con los resultados para el BTC, como se muestra en las Tablas 2 y 3 . En el caso del coeficiente de absorción capilar este tuvo una reducción a medida que iba aumentando el porcentaje de emulsión llegando a una reducción máxima del 97\% aproximadamente para la sustitución del 100\%. Finalmente, la porosidad efectiva no tuvo una variación representativa teniendo solo una reducción del $82 \%$ en relación a la referencia.

Con el fin de verificar la influencia del porcentaje de sustitución de emulsión en el RPA del BTC y de los cilindros de mortero se realizó una correlación de los parámetros estudiados (Figura 3).

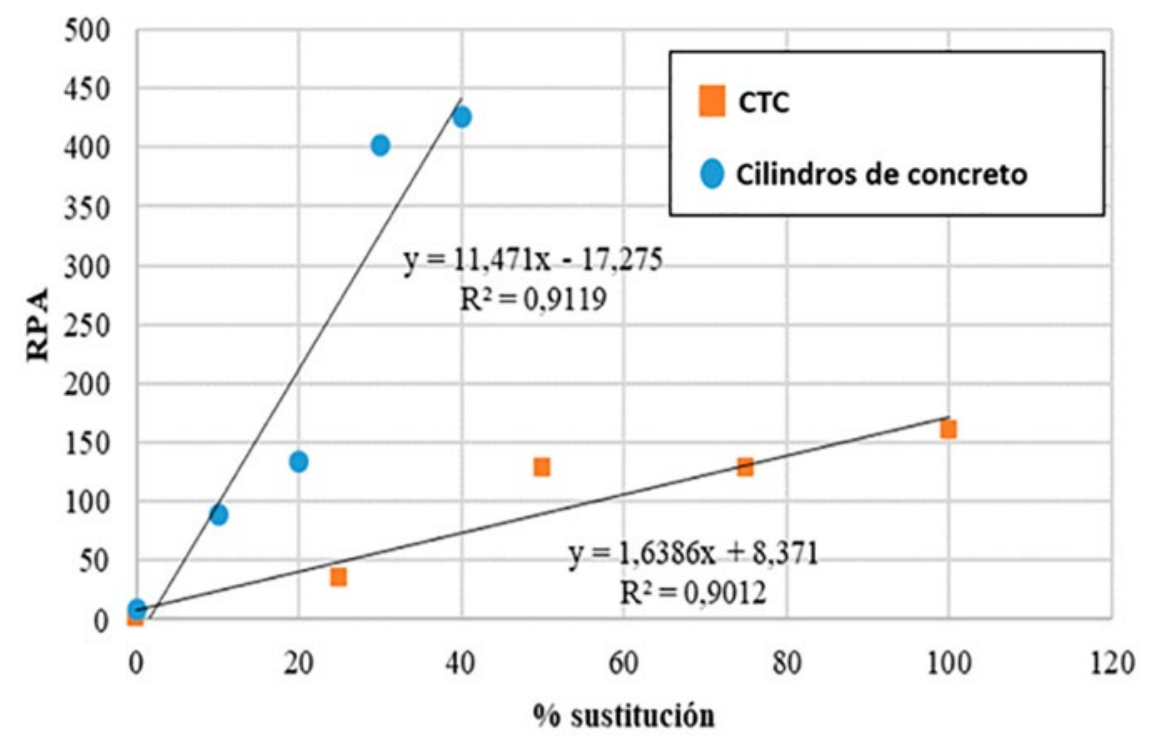

Figura 3. Correlación entre la sustitución y la RPA.

En la Figura 3 se muestra que la sustitución de emulsión es notablemente más efectiva con los cilindros de mortero presentando una tendencia preponderantemente lineal, ya en el caso de los 
CTC mejora el RPA sin embargo su mejoría no llega a ser tan notable ya que la pendiente de su línea de tendencia es notablemente más baja.

De igual forma fue analizada la relación entre el CAC y el porcentaje de sustitución de emulsión para el CTC y los cilindros de mortero (Figura 4).

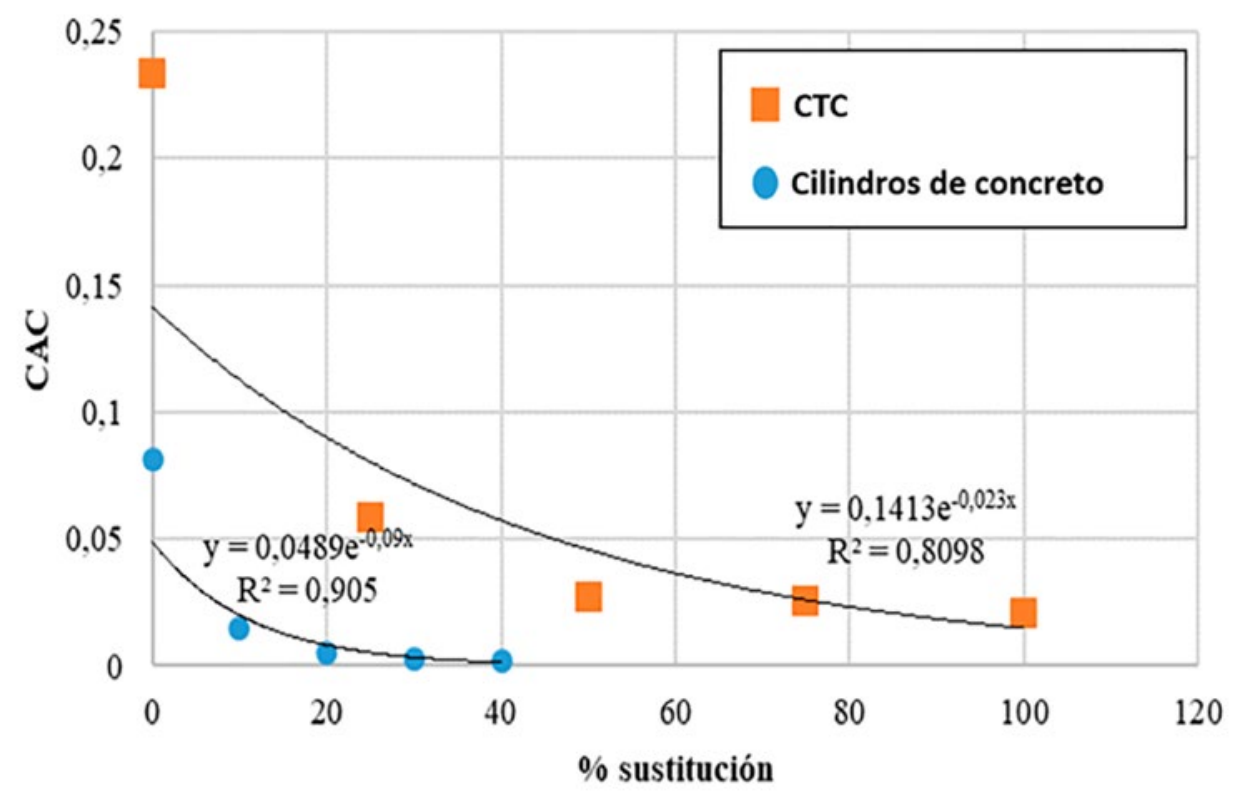

Figura 4. Correlación entre la sustitución y el CAC.

La variación del CAC en función del porcentaje de sustitución de emulsión presenta un comportamiento exponencial para los cilindros de mortero, ya que a medida que aumenta el porcentaje de sustitución de emulsión disminuye de forma notable el CAC con los dos materiales estudiados. La sustitución de emulsión se mostró más efectiva con los cilindros de mortero como fue mostrado en la Figura 5, la pendiente de la línea de tendencia es más elevada cuando es comparada al CTC, llegando a valores de CTC más bajos con un menor porcentaje de emulsión. De igual forma la variación de la porosidad en función del porcentaje de sustitución de emulsión presenta un comportamiento exponencial para los cilindros de concreto como visualizado en la Figura 5. 


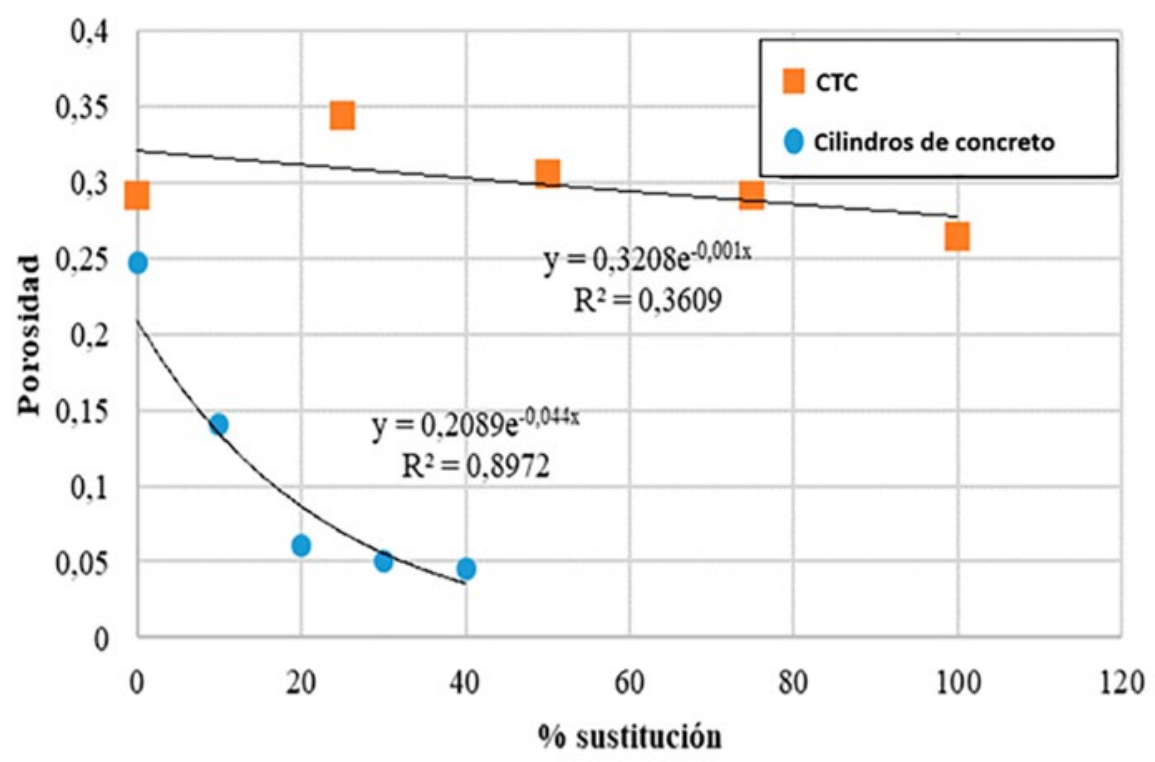

Figura 5: Correlación entre la sustitución y la porosidad.

Dadas las correlaciones mostradas en la Figura 6, las disminuciones más grandes de porosidad se presentaron con los bloques de concreto, esto debido a ser un material más homogéneo cuando comparado al CTC. En el caso d los CTC las disminuciones de porosidad fueron más bajas debido a que es un material más heterogéneo lo que explica la baja efectividad de la reducción de la porosidad.

En la Figura 6 se presentan los boxplot relacionados a CAC, porosidad efectiva y RPA en función de los porcentajes de sustitución de emulsión.
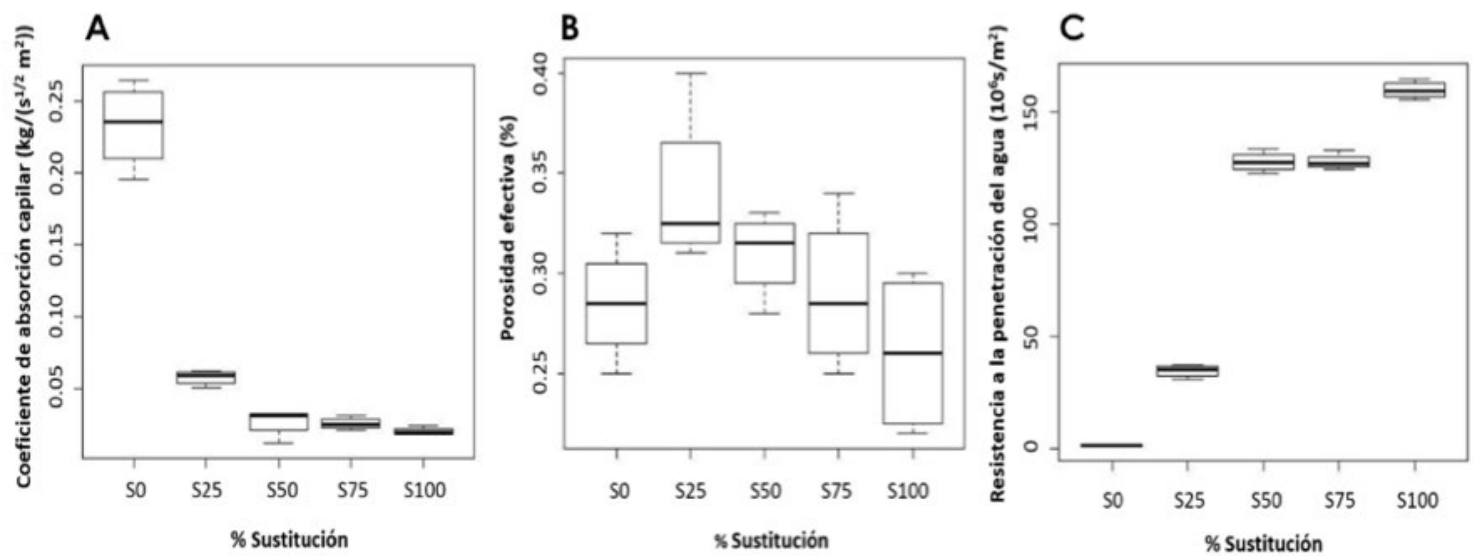

Figura 6: Boxplot: A) CAC, B) Porosidad efectiva y C) RPA.

Como fue mostrado en la Figura 6A, la referencia y la adición de emulsión del 25\% fueron las mezclas que presentaron diferencia significativa con los otros porcentajes analizados. La adición del 50\%, 75\% y 100\% de emulsión no presentaron diferencia significativa entre ellas, por lo tanto, después del $50 \%$ de adición no es tan funcional continuar aumentando la emulsión en el material para reducir el coeficiente de absorción capilar.

En la Figura 6B se muestran los resultados del boxplot de los porcentajes de adición de emulsión y la porosidad efectiva del material. La referencia no presento diferencia significativa con ningún porcentaje de sustitución analizado, luego la adición de emulsión no influencia tanto esta propiedad estadísticamente hablando. 
Finalmente, en la Figura 6C se puede analizar que la referencia presenta diferencia significativa con todos los porcentajes de adición de emulsión analizados al igual que la adición del 25\%. Por otro lado, las adiciones de $50 \%$ y $75 \%$ no presentan diferencia significativa entre ellas.

\section{CONCLUSIONES}

A partir de los resultados obtenidos se puede concluir que el uso de emulsión asfáltica en frío permite mejoras con relación CAC, RPA y porosidad, tanto en cilindros de mortero como en CTC, logrando incrementos porcentuales de $10224,8 \%$ para RPA del CTC con $100 \%$ de emulsión asfáltica y de $4589,03 \%$ para los bloques de concreto con $40 \%$ de aditivos respectivamente; además el uso de la emulsión permite una reducción del CAC del $91.4 \%$ con $100 \%$ de emulsión para los BTC y del $97.5 \%$ para las muestras cilíndricas en mortero con $40 \%$ de aditivo. De igual manera se presentaron mejoras con relación a la reducción de la porosidad en ambos materiales analizados, para el CTC con $100 \%$ se presentó una reducción de $10.3 \%$ y para los bloques de concreto se presentó una reducción del $81.8 \%$ con $40 \%$ de emulsión respectivamente.

Además de lo anterior, fue verificada la correlación proporcional entre el porcentaje de sustitución de emulsión y el RPA para los dos materiales analizados, siendo la sustitución más efectiva siempre en los cilindros de mortero dada la naturaleza más homogénea del material. Por otra parte, la relación de CAC y porcentaje de sustitución de emulsión es inversamente proporcional para los datos analizados, siendo mayormente efectiva esta reducción en los cilindros de mortero.

En el caso de la porosidad, esta fue ampliamente reducida con el aumento de la sustitución de emulsión, las mayores reducciones se presentaron en el caso de los cilindros de mortero, tal hecho es explicado por la homogeneidad del material.

Con relación a los resultados obtenidos entre los dos materiales analizados, tierra y mortero, se determina que el uso de emulsión asfáltica conlleva a la mejora de las características de impermeabilidad de ambos materiales, lo que nos permite establecer que el uso de compuestos bituminosos pude contribuir al incremento de la durabilidad de los sistemas de mampostería; es por esta razón que independiente del tipo de construcción aplicada, bien sea tradicional, donde los morteros juegan un gran papel en los sistemas constructivos ó construcción vernácula donde la tierra es uno de los principales materiales utilizados, la humedad en ambas tipologías de construcción genera deterioro, por lo siguiente los compuestas bituminosos se convierten en una alternativas eficiente a la solución de problemas ligados a las humedades debidas a la permeabilidad y porocidad de materiales, logrando una mayor eficiencia este aditivo bituminoso en los morteros sin menospreciar su uso con relación a la tierra compactada.

Para futuras investigaciones se recomienda analizar el uso de las emulsiones asfálticas como reductores de la presencia de eflorescencias en bloques de concreto y BTC (Bloques de tierra comprimida), lo anterior como elemento de mejora de la durabilidad de mampuestos para sistemas de mampostería.

\section{REFERENCIAS}

Adam E. and Agib, A. (2001) "Compressed Stabilised Earth Block Manufacture in Sudan", United Nations Educational, Scientific and Cultural Organization, Paris, Francia.

Almusallam, A. A. Khan, F. M. Dulaijan, S. U. and Al-Amoudi, O. S. (2002) Effectiveness of surface coatings in improving concrete durability, Cement and Concrete Composites, 473-481. DOI: https://doi.org/10.1016/S0958-9465(02)00087-2. 
American Society for Testing and Materials (ASTM), C 1585-04. (2007) Standard test method for measurement of rate of absorption of water by hydraulic-cement concretes, ASTM Annual book of standards, West Conshohocken, Pa.

American Society for Testing and Materials (ASTM), D 422. (2007) Standard test method for particle - Size analysis of soils, ASTM Annual book of standards. West Conshohocken, Pa

Anastasopoulos, I. (2013) Building damage during nearby construction: Forensic analysis, Engineering Failure Analysis, 34:252-267.

DOI: https://doi.org/10.1016/j.engfailanal.2013.08.003.

Badogiannis, E. G. Sfikas, I. P. Voukia, V. D. Trezos, K. G. and Tsivilis, S. G. (2015) Durability of metakaolin Self-Compacting Concrete, Construction and Building Materials, 82:133-141. DOI: https://doi.org/10.1016/j.conbuildmat.2015.02.023.

Bai, J. (2016) “Durability of sustainable construction materials”, Second Edition. Elsevier Ltd.

Bołtryk, M. and Małaszkiewicz, D. (2013) Application of anionic asphalt emulsion as an admixture for concrete, Construction and Building Materials, 40:556-565.

DOI: https://doi.org/10.1016/j.conbuildmat.2012.11.110.

Broto, C. and Soria, V. (2005) “Enciclopedia Broto de patologías de la construcción”, 6, Structure , Madrid, España.

Cañola, H. and Echavarría, C. (2017) Concrete blocks with paraffin wax, Lámpsakos, 17:14-19. DOI: http://dx.doi.org/10.21501/21454086.2346.

Cañola, H., Granda-Ramírez, F., \& Arroyave-Rojas, J. (2020). Emulsión asfáltica como alternativa de reducción de la presencia de eflorescencias en morteros de pega. Revista UIS Ingenierías, 20(1), 103-114. DOI: https://doi.org/10.18273/revuin.v20n1-2021009

Cañola, H. D. Builes-Jaramillo, A. Medina, C. A. and González-Castañeda, G. E. (2018) Bloques de Tierra Comprimida (BTC) con aditivos bituminosos bitumen emulsión, TecnoLógicas, 21(43):135-145. DOI: https://doi.org/10.22430/22565337.1061.

Carrer, P. Wargocki, P. Fanneti, A. Bischof, W. De Oliveira, E. Hartmann, T. Ke'halopoulos, S. Palkonen, S. and Seppänen, O. (2015) What does the scientific literature tell us about the ventilation e health relationship in public and residential buildings?, Building and Environment, 94 (1):273-286. DOI: https://doi.org/10.1016/j.buildenv.2015.08.011.

Cho, B. Park, D. Kim J. and Hamasaki, H. (2017) Study on the heat-moisture transfer in concrete under real environment, Construction and Building Materials, 132:124-129.

DOI: https://doi.org/10.1016/j.conbuildmat.2016.11.121.

Cui, C. Yuan, Z. and Li, Y. (2012) Fly-Ash Usage in New Cement-Based Material for Concrete Waterproofing, Advanced Materials Research, 535-537:1902-1906.

DOI: https://doi.org/10.4028/www.scientific.net/AMR.535-537.1902. 
Falchi, L. Müller, U. Fontana, P. Izzo F. C. and Zendri, E. (2013) Influence and effectiveness of water-repellent admixtures on pozzolana-lime mortars for restoration application, Construction and Building Materials, 49: 272-280. DOI: https://doi.org/10.1016/j.conbuildmat.2013.08.030.

Jiménez López, L. (2005) “Humedades en la construcción”, CEAC, Barcelona, España.

Karagiannis, N. Karoglou, M. Bakolas, A. Krokida M. and Moropoulou, A. (2017) Drying kinetics of building materials capillary moisture, Construction and Building Materials, 137: 441-449. DOI: https://doi.org/10.1016/j.conbuildmat.2017.01.094.

Lanzón M. and García-Ruiz, P. A. (2009) Evaluation of capillary water absorption in rendering mortars made with powdered waterproofing additives, Construction and Building Materials, 23(10):3287-3291. DOI: https://doi.org/10.1016/j.conbuildmat.2009.05.002.

Litvan, G. G. (1996) Waterproofing of parking garage structures with sealers and membranes: the Canadian experience, Construction and Building Materials, 10(1):95-100.

DOI: https://doi.org/10.1016/0950-0618(95)00061-5.

Monjo Carrió, J. and Maldonado Ramos, L. (2001) "Patología y técnicas de intervención en estructuras arquitectónicas”, MUNILLALER, Barcelona, España.

Muhammad, N. Z. Keyvanfar, A. Zaimi, M. Majid, A. Shafaghat, A. and Mirza, J. Waterproof performance of concrete: A critical review on implemented approaches, Construction and Building Materials, 101:80-90. DOI: https://doi.org/10.1016/j.conbuildmat.2015.10.048.

Othman, N. L. Jaafar, M. Harun W. M. and Ibrahim, F. (2015) A Case Study on Moisture Problems and Building Defects, Procedia - Social and Behavioral Sciences, 170:27-36. DOI: https://doi.org/10.1016/j.sbspro.2015.01.011.

Pereira, C. De Brito, J. and Silvestre, J. D. (2018) Contribution of humidity to the degradation of façade claddings in current buildings, Engineering Failure Analysis, 90:103-115. DOI: https://doi.org/10.1016/j.engfailanal.2018.03.028.

Saija, L. M. (1995). Waterproofing of portland cement mortars with a specially designed polyacrylic latex, Cement and Concrete Ressearch. 25(3):503-509. DOI: https://doi.org/10.1016/0008-8846(95)00039-F.

Shoemaker, R. C. and House, D. E. Sick building syndrome (SBS) and exposure to water-damaged buildings: Time series study, clinical trial and mechanisms, "Neurotoxicology and Teratology", 28:573-588. DOI: https://doi.org/10.1016/j.ntt.2006.07.003.

Silva, E. F. Morerira, M. Manzano, M. A. and Blanco, R. (2017) Case study of permeabilityreducing admixture use in anti-flotation slabs: building in Brasilia, Brazil, Journal of Building Pathology and Rehabilitation, 2 (1):1- 9. 2017. DOI: https://doi.org/10.1007/s41024-016-0014-5.

Teng, L. Huang, R. Chen, J. Cheng A. and Hsu, H. (2014) A Study of Crystalline Mechanism of Penetration Sealer Materials, Materials, 7(1):399-412. DOI: https://doi.org/10.3390/ma7010399. 
Tokimatsu, K. Tamura, S. Suzuki H. and Katsumata, K. (2012) Building damage associated with geotechnical problems in the 2011 Tohoku Pacific Earthquake, Soils and Foundations, 52(5): 956974. DOI: https://doi.org/10.1016/j.sandf.2012.11.014.

Tsukagoshi, M. Miyauchi, H. and Tanaka, K. (2012) Protective performance of polyurethane waterproofing membrane against carbonation in cracked areas of mortar substrate, Construction and Building Materials, 36: 895-905. DOI: https://doi.org/10.1016/j.conbuildmat.2012.06.072.

UNE: PrUNE 83.982 (2007). Durabilidad del hormigón. Determinación de la absorción de agua por capilaridad del hormigón endurecido, Método Fagerlund.

Wilson, M. A. Hoff, W. D. and Hall, C. (1994) Water movement in porous building materialsXI. Capillary absorption from a hemispherical cavity, Building and Environment, Building and the Environment, 29(1):99-104. DOI: https://doi.org/10.1016/0360-1323(94)90058-2.

Zhu, Y. Kou, S. Poon, C. Dai, J. and Li, Q. (2013) Cement \& Concrete Composites Influence of silane-based water repellent on the durability properties of recycled aggregate concrete, Cement and Concrete Composites, 35(1):32-38.

DOI: https://doi.org/10.1016/j.cemconcomp.2012.08.008.

Zürcher, C. and Frank, T. (2014) "Physique du bâtiment: construction et énergie", 1st ed. vdf Hochschulvlg AG an der ETH Zürich. Zürich. Alemania. 